\title{
Adaptação de um protocolo para avaliação do bem-estar de cães (Canis familiaris) da Polícia Militar
}

\author{
Adaptation of a protocol for the evaluation of welfare of military \\ dogs (Canis familiaris)
}

Amanda Macedo da Silva, Aline Cristina Sant'Anna

Universidade Federal de Juiz de Fora (UFJF), Juiz de Fora, MG, Brasil

\section{Resumo}

Com o presente estudo objetivou-se propor um protocolo para a avaliação do bem-estar de cães utilizados em atividades policiais, a partir de uma adaptação do protocolo Shelter Quality (SQ Barnard et al., 2014), visando fornecer uma ferramenta para mensuração da qualidade de vida destes animais. Participaram do estudo 11 cães pertencentes ao canil do Pelotão de Policiamento com Cães da 4ํㅡㄹ Cia PM Independente de Policiamento Especializado, Juiz de Fora, MG. 0 estudo foi dividido em: a) adaptação do protocolo SQ para avaliação do bem-estar dos cães; b) descrição da rotina de trabalho dos cães. Os indicadores de bem-estar presentes no protocolo SQ foram organizados em três níveis de registro: medidas tomadas para o canil (tipo de alojamento, frequência de exercício, taxas de mortalidade e morbidade, manejo alimentar), medidas nos boxes (espaço disponível, tipo de cama, presença de superfície pontiaguda, fornecimento de água, conforto térmico e nível de latidos) e medidas obtidas nos animais, considerando cada indivíduo como uma unidade amostral (condição corporal, limpeza dos animais, condição do tegumento, claudicação, problemas respiratórios, evidência de dor, diarreia, comportamento anormal, reação ao ser humano e estados emocionais). Por tratar-se de uma pesquisa metodológica e descritiva, os dados foram analisados de modo predominantemente qualitativo. De acordo com os resultados, o protocolo SQ foi aplicável e ofereceu informações relevantes sobre o bem-estar dos cães. No entanto, o mesmo foi considerado incompleto, sendo necessária a inclusão de indicadores adicionais para que contemplasse um maior número de pontos críticos do bem-estar de cães de trabalho; são eles: manejo sanitário, transporte dos cães, higiene dos boxes, disponibilidade de enriquecimento ambiental, local de permanência dos cães durante a limpeza e manutenção dos boxes e socialização dos cães. Quanto à rotina dos cães, a carga horária de policiamento variou de $2 \mathrm{~h}$ a $10 \mathrm{~h}$ de trabalho semanal/ animal, variando de 0 a 4 saídas semanais para policiamento, além de 0 a 160 minutos semanais de recreação fora dos boxes. Embora tenha sido elaborado para avaliação do bem-estar de cães de abrigo, o protocolo SQ foi exequível e prático para a avaliação de cães de trabalho militar. Foi necessário, porém, realizar algumas adaptações para sua melhor adequação às condições de canis militares.

Palavras-chave: Animais domésticos. Cães de trabalho. Comportamento animal. 


\section{Abstract}

The present study aimed to propose a protocol for the evaluation of the welfare of military dogs by developing an adaptation of the Shelter Quality Protocol to provide a practical tool to assess dogs' quality of life. Eleven dogs from the Pelotão de Policiamento com Cães da 4a Cia PM Independente de Policiamento Especializado, Juiz de Fora, Brazil, were used. The study was divided into two approaches: a) adaptation of the SQ protocol to evaluate the welfare ofdogs; b) description of the dogs' working routine. In the SQ protocol, welfare measures were taken according to three scoring levels: measures for the kennel (housing type, frequency of exercise, mortality and morbidity rates, food management), measures at the pens (space allowance, pen hygiene, bedding, presence of sharp edges, water supply, thermal comfort and barking level) and individual measures, considering each animal as a unit (body condition, dog cleanliness, skin condition, lameness, respiratory disorders - coughing, evidence of pain, diarrhea, abnormal behavior, reaction toward humans and emotional states). Data was analyzed in a qualitative manner, since it was a methodological and descriptive study. According to the results, the SQ protocol was feasible and provided relevant information about the welfare of military dogs. However, the original protocol was considered incomplete, since it did not address all critical points of the welfare of working dogs in kennels. Therefore, it was necessary to include additional indicators to gather a greater number of relevant aspects of animal welfare, such as: health management, dog transportation, body hygiene, environmental enrichment, place for dog maintenance while cleaning the boxes, and socialization of dogs. Regarding the dogs' routine, the policing hours ranged from 2 to 10 working hours per week per animal, with 0 to 4 weekly outings per animal. The dogs also spent 0 to 160 minutes per week in outdoor recreational activities and exercise. Although the $S Q$ protocol has been developed to evaluate the welfare of shelter dogs, it was feasible and practical for the evaluation of military dogs' welfare. However, in order to better suit the conditions of military kennels, some adaptations were necessary.

Keywords: Domestic animals. Working dogs. Animal behavior.

\section{Introdução}

Há tempos os cães vêm exercendo um importante papel em nossa sociedade, trazendo diversos benefícios para o ser humano, como o auxílio em atividades de trabalho, proteção e companhia (Broom e Fraser, 2010). Assim, os cães passaram a ter uma interação tão estreita com o ser humano como nunca atingida com qualquer outra espécie de animal doméstico, o que foi possível graças às suas habilidades sociais e cognitivas únicas (Li et al., 2014), permitindo que desenvolvessem tarefas complexas, como aquelas inerentes ao trabalho militar. Nota-se um crescente aumento no interesse científico em entender diversos aspectos da interação entre ser humano e cão, incluindo também estudos sobre os cães de trabalho militar (Haverbeke et al., 2010a,b). Esses cães são treinados para exercer diversas tarefas, como, por exemplo, a identificação de indivíduos, busca ativa por narcóticos, explosivos, substâncias químicas em geral, dentre outras (Burghardt Jr, 2003; Haverbeke et al., 2008a).

Não é incomum que os cães de trabalho recebam menos atenção se comparados aos cães de companhia, sendo por vezes considerados uma ferramenta de trabalho, o que pode colocar seu bem-estar em risco (Schilder e van der Borg, 2004). Quanto aos trabalhos realizados pelos cães, é comum que as atividades policiais submetam esses animais a situações potencialmente estressantes, tais como ruído excessivo, condições climáticas extremas, exaustão corporal e mental, novidades, imobilização, necessidade de transporte frequente e manejos inadequados (Beerda et al., 1997; Baker e Miller, 2013; Diverio et al., 2016). Além disso, estes cães passam a maior parte de suas vidas confinados, fazendo com que as condições de alojamento sejam cruciais para garantir níveis aceitáveis de bem-estar animal. Portanto, percebe-se a necessidade de iniciativas tanto do ponto de vista da pesquisa científica, quanto do estabelecimento de regulamentações e normativas que tenham como foco a melhoria da qualidade de vida dos cães no cenário do serviço militar.

Alguns estudos prévios tiveram como objetivo a avaliação de diversos fatores relacionados ao bem-estar de cães de trabalho militar, incluindo a qualidade da interação humano animal (Lefebvre et al., 2007), os efeitos benéficos do contato social com coespecíficos (Gfrerer et al., 2018) e da disponibilidade de enriquecimento ambiental e 
alimentar (Gaines et al., 2008; Lefebvre et al., 2009). Foram investigados também os efeitos de diferentes tipos de estressores ambientais sobre as respostas comportamentais e fisiológicas de cães de trabalho militar, como, por exemplo, o efeito de objetos novos e ruídos abruptos (Haverbeke et al., 2008a), perturbação por instalação de equipamentos em seus boxes (Lefebvre et al., 2010) e condições ambientais extremas (Baker e Miller, 2013; Diverio et al., 2016).

Outro aspecto previamente investigado foi o efeito de diferentes tipos de treinamento sobre o comportamento e a performance do cão (Haverbeke et al., 2008b, 2010a), além dos benefícios da familiarização prévia e inserção gradual do animal na rotina de trabalho do canil (Rooney et al., 2007; Haverbeke et al., 2010b). No Brasil, os poucos estudos publicados sobre o bemestar de cães militares tiveram como objetivo avaliar o estresse provocado pelo trabalho e seus impactos na performance reprodutiva dos cães (Baptista Sobrinho, 2009; Arcuri, 2015), sendo também investigado o estresse causado pelo confinamento em caixas de transporte e seus efeitos no desempenho dos cães em atividades de busca e detecção de drogas e explosivos (Machado, 2013).

Embora os diversos estudos prévios tenham abordado efeitos de alguns fatores sobre o bemestar dos cães de trabalho militar, são escassas as publicações com enfoque mais geral, incluindo a avaliação de elementos do próprio animal e do ambiente de criação (Rooney et al., 2009). Não foram encontrados na literatura técnico-científica protocolos de avaliação do bem-estar dos cães de trabalho militar que contemplem uma investigação integrativa de aspectos do ambiente com elementos individuais dos próprios cães, capazes de expressar sua saúde física e mental a partir dos princípios relacionados à boa alimentação, bom alojamento, boa saúde e comportamento apropriado.

Com o presente estudo objetivou-se propor um protocolo para a avaliação do bem-estar de cães utilizados em atividades policiais a partir de uma adaptação do protocolo Shelter Quality (Barnard et al., 2014), visando, assim, fornecer uma ferramenta para a mensuração da qualidade de vida destes animais, permitindo a melhoria do seu bem-estar e desempenho durante o trabalho militar.

\section{Material e métodos}

0 presente estudo foi realizado no canil do Pelotão de Policiamento com Cães da 4 a Cia PM Independente de Policiamento Especializado, Juiz de Fora, MG, no período de agosto a novembro de 2017. A pesquisa foi aprovada pela Comissão de Ética no Uso de Animais da Universidade Federal de Juiz de Fora, Protocolo 039/2017. Foram utilizados 11 cães (quatro machos e sete fêmeas) com idade variando de 1,7 a 10 anos, das raças Pastor Alemão $(n=7)$ e Pastor Belga Malinois $(n=4)$, sendo este o número total de animais disponíveis no canil no momento da pesquisa (Tabela 1).

Tabela 1 - Descrição de todos os cães que participaram da pesquisa $(n=11)$

\begin{tabular}{ccccc}
\hline Cão $^{*}$ & $\begin{array}{c}\text { Idade } \\
\text { (anos) }\end{array}$ & Sexo & Raça & Especialidade \\
\hline A & 3,0 & Macho & Pastor Alemão & FE \\
Ba & 7,0 & Macho & Pastor Alemão & FE \\
Bi & 4,0 & Fêmea & Pastor Alemão & FE \\
C & 10,0 & Fêmea & Pastor Belga Malinois & FB \\
E & 7,0 & Fêmea & Pastor Alemão & FE \\
F & 8,0 & Fêmea & Pastor Belga Malinois & FB \\
la & 6,0 & Fêmea & Pastor Alemão & FE \\
I & 1,7 & Fêmea & Pastor Alemão & FB \\
K & 6,0 & Fêmea & Pastor Alemão & FE \\
P & 10,0 & Macho & Pastor Belga Malinois & FB \\
T & 7,0 & Macho & Pastor Belga Malinois & FB \\
\hline & & & &
\end{tabular}

Nota: * Letras representam as iniciais dos nomes de cada cão. FE = faro de entorpecentes. $\mathrm{FB}$ = faro para busca e captura de pessoas.

Os cães apresentavam especialidades variadas, sendo treinados para faro de entorpecentes e para busca e captura de pessoas, conforme caracterizados no próprio canil. Para a avaliação do bem-estar animal foram aplicados indicadores baseados em uma adaptação do Shelter Quality Protocol (Barnard et al., 2014), desenvolvido para avaliação do bem-estar de cães em abrigos. Assim, o presente estudo foi dividido em: a) adaptação do protocolo Shelter Quality (SQ) para avaliação do bem-estar dos cães; b) descrição da rotina de trabalho dos 
cães, baseada em informações fornecidas pelos responsáveis pelo canil.

\section{Adaptação do protocolo Shelter Quality para avaliação do bem-estar dos cães}

Foi realizada a aplicação do protocolo Shelter Quality (Barnard et al., 2014), buscando adequá-lo às condições do presente estudo, conforme descrito a seguir. Os indicadores de bem-estar animal incluídos no protocolo original são organizados em três níveis de registro: i) medidas tomadas para o canil, ii) medidas tomadas nos canis individuais (aqui referidos como boxes) e iii) medidas obtidas nos animais, registradas individualmente para cada cão (Tabela 2). As medidas para o canil foram obtidas por meio de relatos dos responsáveis pelo estabelecimento, enquanto as medidas dos boxes e dos animais foram registradas pela própria equipe da pesquisa.

A adaptação consistiu em identificar, durante a aplicação do Protocolo SQ pontos críticos de bem-estar dos cães de trabalho que não estavam contemplados no protocolo, ou seja, não havia qualquer indicador tratando de acessá-los. Estes foram, então, incluídos no protocolo original, conforme descrito nos resultados do presente estudo.

Tabela 2 - Descrição das medidas utilizadas para avaliação do bem-estar dos cães de trabalho militar

\begin{tabular}{|c|c|}
\hline Medida & Descrição \\
\hline \multicolumn{2}{|c|}{ Medidas obtidas para o canil } \\
\hline Alojamento & Número de boxes individuais e coletivos (número de cães/boxe). \\
\hline Exercício & $\begin{array}{l}\text { Frequência de liberação em área externa ou caminhada na guia: } 1 \text { (diária), } 2 \text { (semanal) e } 3 \text { (não ocorre ou não } \\
\text { há uma rotina regular). }\end{array}$ \\
\hline Taxa de morbidade & Número de tratamentos clínicos por animal/ano. \\
\hline Taxa de mortalidade & Número de animais que sofreram eutanásia/ano e óbitos/ano. \\
\hline Alimentação & $\begin{array}{l}\text { Regime de alimentação (número de vezes/dia), tipo de alimento oferecido e existência de dieta especial para } \\
\text { filhotes, doentes e senis. }\end{array}$ \\
\hline \multicolumn{2}{|c|}{ Medidas obtidas nos boxes } \\
\hline Espaço disponível & 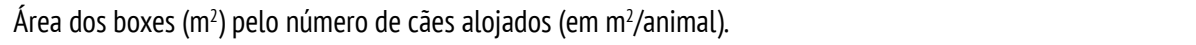 \\
\hline Higiene dos boxes & Escore visual: 1 (limpo), 2 (sujo) e 3 (muito sujo). \\
\hline Tipo de cama & $\begin{array}{l}\text { Descrição do tipo de cama e sua adequação, em escores: } 1 \text { (adequada) e } 2 \text { (inadequada; quando há menos } \\
\text { de uma cama por cão, se oferece algum tipo de risco para o animal e/ou se está úmida e com fezes). }\end{array}$ \\
\hline Superfície pontiaguda & Presença no interior dos boxes: 0 (ausente) e 1 (presente). \\
\hline Fornecimento de água & Tipo, funcionamento adequado, condições de higiene e segurança oferecida pelos bebedouros. \\
\hline Conforto térmico & Número de animais ofegantes ou com tremor. \\
\hline Nível de latidos & Número de cães que ladram na presença do avaliador durante 1 minuto de observação. \\
\hline \multicolumn{2}{|c|}{ Medidas obtidas nos animais } \\
\hline Condição corporal & $\begin{array}{l}\text { Em escores: } 1 \text { (muito magro: os ossos são facilmente visíveis nas costelas, cintura pélvica e vértebras lombares, } \\
\text { apresentando perda de massa muscular, cintura acentuada e flacidez abdominal); } 2 \text { (aceitável: nenhum excesso } \\
\text { de gordura, com cintura observável quando vista de cima e abdômen aprofundado quando visto de lado); } \\
3 \text { (muito gordo: camada de gordura excessiva, costelas não palpáveis, depósito de gordura no pescoço e } \\
\text { membros, ausência de cintura e distensão abdominal presente). }\end{array}$ \\
\hline Condição do tegumento & Número de animais com evidências de ferimentos, perda de pelos, inchaços e ectoparasitas. \\
\hline Claudicação & $\begin{array}{l}\text { Registra-se em escores, após observar o cão caminhar: } 1 \text { (normal), } 2 \text { (apresenta ritmo irregular no passo), } \\
3 \text { (severamente afetado, incapaz de se locomover). }\end{array}$ \\
\hline Problemas respiratórios & Número de animais com evidência de tosse. \\
\hline Evidência de dor & $\begin{array}{l}\text { Número de animais com sinais de dor (irresponsivos, apáticos, deitados, evitam contato, rosnam quando há } \\
\text { tentativa de aproximação). }\end{array}$ \\
\hline
\end{tabular}


Tabela 2 - Descrição das medidas utilizadas para avaliação do bem-estar dos cães de trabalho militar

(continuação)

\begin{tabular}{|c|c|}
\hline Medida & Descrição \\
\hline Evidência de diarreia & Fezes líquidas no interior do boxe: 0 (ausente), 1 (presente). \\
\hline Comportamento anormal & $\begin{array}{l}\text { Número de cães com estereotipias (andar em círculo; pacing; girar; saltar na parede) ou outros comportamentos } \\
\text { compulsivos (automutilação, comportamentos direcionados ao ambiente). }\end{array}$ \\
\hline Reação ao ser humano & $\begin{array}{l}\text { É realizada uma avaliação individual de cada cão. } 0 \text { observador aproxima-se de cada boxe, permanece em } \\
\text { silêncio e imóvel por } 30 \text { segundos diante da grade do boxe, observando o comportamento do animal. Em } \\
\text { seguida, o registro é realizado em escores: } 0 \text { (sem sinais de medo ou agressão e postura neutra: permanece } \\
\text { relaxado, olhando para ou ignorando o avaliador, amigável/sociável, aproxima-se a curtas distâncias ou tenta } \\
\text { interagir com o avaliador); } 1 \text { (sem sinal de medo ou agressão e postura atenta/alerta: permanece a uma certa } \\
\text { distância e não tenta interagir com o avaliador); } 2 \text { (medo sem sinais de agressão: geralmente permanece a } \\
\text { longas distâncias ou foge do avaliador, se esquiva, com posturas de submissão, orelhas para trás, evita contato } \\
\text { ocular direto com o avaliador, cauda abaixada ou posicionada entre as pernas); } 3 \text { (medo com sinais de agressão: } \\
\text { postura defensiva, posterior do corpo abaixado, pêlos das costas arrepiados, cauda abaixada e tensa ou entre } \\
\text { as pernas, orelhas para trás, pupila dilatada, focinho tenso e enrugado, expondo os dentes); } 4 \text { (agressão sem } \\
\text { sinais de medo: postura ofensiva, corpo direcionado para a frente, pêlos podem estar arrepiados ou não, cauda } \\
\text { erguida, orelhas levantadas, contato ocular direto com o avaliador, lábios curvados, mostrando os dentes). }\end{array}$ \\
\hline Estado emocional & 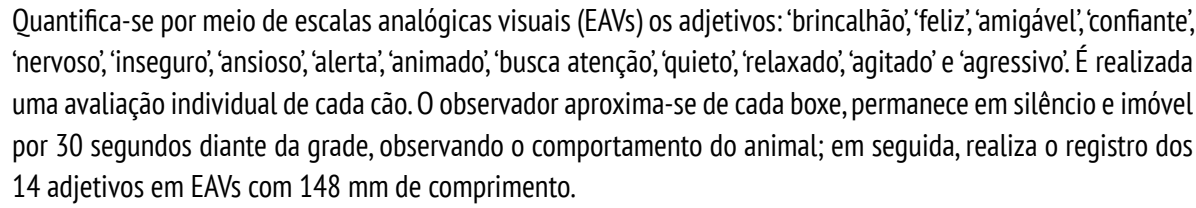 \\
\hline
\end{tabular}

Nota: Adaptado de Barnard et al., 2014.

\section{Descrição da rotina dos cães}

Ao longo de seis semanas foi realizada uma avaliação da rotina diária dos cães a fim de estimar a carga horária semanal de trabalho à qual os cães são submetidos e como esta é distribuída entre os animais em atividade. Para isso, foi solicitado aos próprios policiais atuantes no canil que registrassem os horários de saída e chegada dos cães para as seguintes atividades: treinamento, policiamento e recreação fora dos boxes.

\section{Análise dos dados}

Por se tratar de uma pesquisa com abordagem metodológica e descritiva, os dados foram sintetizados, analisados e apresentados de modo predominantemente qualitativo. Quando pertinente, foram realizadas análises estatísticas descritivas, com obtenção de médias para as variáveis contínuas e distribuição das notas para as variáveis categóricas utilizando as porcentagens dos escores.
Para análise dos dados da QBA foi aplicada estatística multivariada, usando-se o método da Análise de Componentes Principais (ACP). A ACP é um tipo de método que reúne variáveis em uma matriz de dados "p" e encontra combinações destas para produzir índices (os componentes principais) que descrevam a variação dos dados (Manly, 2008). Para esta análise, as variáveis devem ser correlacionadas e, sendo assim, um grande número de variáveis originais pode ser adequadamente representado por dois ou três componentes principais. Para a avaliação do estado emocional dos cães foram utilizados 14 adjetivos predefinidos, que permitiram ao observador quantificá-los a partir da observação das expressões comportamentais dos cães, ou seja, sua linguagem corporal. Os adjetivos foram quantificados em uma escala analógica visual com $148 \mathrm{~mm}$ de comprimento. As notas de cada animal, em cada termo, foram obtidas medindo-se a distância em milímetros desde o início da escala até a marca feita pelo observador, sendo que o valor mínimo $(0 \mathrm{~mm})$ representava a ausência da expressão e o valor máximo (148 mm), a manifestação de 
sua mais intensa expressão. A matriz gerada foi composta por 10 animais (linhas) e 14 termos (colunas), sendo a ela aplicada a ACP, a qual gerou três componentes principais (CP) com autovalores acima de 1 . Adjetivos com cargas acima de 0,70 em cada CP foram considerados nos resultados.

\section{Resultados}

\section{Adaptação do protocolo Shelter Quality para avaliação do bem-estar dos cães}

\section{Medidas obtidas para o canil}

Essas informações foram obtidas por meio de relatos dos responsáveis pelo canil. Todos os animais avaliados eram alojados em canis individuais (boxes), sendo liberados para dois tipos de atividades ao ar livre: treinamento e recreação fora dos boxes. Segundo os relatos prestados sobre a rotina de exercício, o período e frequência semanal seguia a escala de trabalho do policial responsável, recebendo escore 3 (i.e., sem rotina regular).

No que diz respeito às taxas de morbidade, segundo as informações do responsável pelo canil, não foi relatada nenhuma ocorrência nos últimos 12 meses. Quanto à mortalidade, foi relatado apenas um caso de eutanásia por problemas de saúde nos últimos 5 anos e nenhum no último ano.

O tipo de alimento ofertado aos animais foi ração seca do tipo super premium, sob venda exclusiva para criadores profissionais, com $10 \%$ de umidade, $26 \%$ de proteína bruta, $16 \%$ de estrato etéreo, $2 \%$ de matéria fibrosa e $6,5 \%$ de matéria mineral. Eram oferecidas duas porções de 300 a 400 g/animal. Eram também oferecidas dietas especiais para filhotes e para animais doentes. Não havia dieta especial para animais idosos.

Além das medidas propostas no protocolo, sentiu-se a necessidade de incluir mais informações relacionadas ao manejo sanitário e condições de transporte dos cães. Constatou-se que o canil não dispunha de médico veterinário próprio, sendo os cães atendidos por profissionais de clínicas particulares. Não havia boxes hospitalares nem boxe maternidade disponíveis, porém animais doentes eram separados. Em relação aos procedimentos em caso de sinais clínicos de displasia coxofemoral, os responsáveis informaram que os cães são avaliados antes de ingressar no serviço militar. Foi constatado o registro por escrito do calendário anual de vacinação dos cães, incluindo vacinas polivalente e antirrábica, além de vermifugação duas vezes ao ano e quando necessário. Além disso, foi relatada a presença de medida para prevenção de problemas dentários, bem como procedimentos operacionais padrão para monitoramento sanitário e presença de protocolo sanitário por escrito.

Por fim, foram registradas também as condições de transporte às quais os cães eram submetidos regularmente para o trabalho militar. 0 compartimento da viatura destinado para o abrigo dos cães apresentava um tablado onde o cão se acomodava durante o trajeto, sendo transportados em compartimentos individuais. Foi avaliado também se as caixas tinham espaço suficiente para que os cães pudessem deitar e girar em torno de seu próprio eixo. Foi relatado pelos responsáveis do canil que durante as operações era fornecida água para os animais e, em casos de operações de longa duração, levava-se também ração.

\section{Medidas obtidas nos boxes}

Tais registros foram obtidos pela equipe da pesquisa. Os canis individuais (boxes) dispunham de $9,80 \mathrm{~m}^{2}$ de área total divididos em área externa $\left(7,34 \mathrm{~m}^{2}\right)$ e interna $\left(2,46 \mathrm{~m}^{2}\right.$ e $2,53 \mathrm{~m}$ de altura), sendo posicionados lado a lado no canil. As grades laterais da área externa tinham $2 \mathrm{~m}$ de altura. As camas eram do tipo "tablado plástico", com dimensões de $0,99 \mathrm{~m}$ por $0,6 \mathrm{~m}$, perfazendo $0,6 \mathrm{~m}^{2} /$ animal, sendo categorizadas como 'adequadas'. Não foram encontradas superfícies pontiagudas. A água era oferecida em recipientes plásticos, com limpeza diária dos bebedouros pela manhã. No momento da avaliação, nenhum dos animais apresentou sinais de desconforto térmico, ou seja, não se mostraram ofegantes ou com tremor. Quanto ao nível de latidos, um animal encontrava-se em policiamento no momento da avaliação. Dentre os cães presentes, $50 \%(5 / 10)$ ladraram frente à presença do avaliador e os demais não apresentaram tal comportamento.

Foi incluída uma avaliação da higiene dos boxes, sendo que $100 \%$ deles se encontravam limpos 
(escore 1), sem presença de acúmulo de fezes e urina no momento da visita. Além disso, o protocolo SQ não faz referência à disponibilidade de itens de enriquecimento ambiental, o que foi incluído na presente avaliação. Não foi diagnosticado o oferecimento de qualquer item de enriquecimento para os cães, como brinquedos, itens para morder ou manipular, itens alimentares como petiscos ou ossos, nem itens de enriquecimento sensorial.

Por fim, sentiu-se a necessidade da inclusão de informações sobre o manejo do cão durante a limpeza e manutenção dos boxes. Foi observado que o tempo médio de limpeza de cada boxe variava de 10 a 20 minutos diários. Algumas vezes a limpeza era realizada com o animal dentro do próprio boxe, interagindo com os cuidadores. Em outros casos os animais eram soltos individualmente no centro de treinamento (CT) ou ficavam soltos pelas dependências do canil. O CT não dispunha de abrigos contra intempéries, nem havia disponibilidade de água para os animais.

\section{Medidas nos animais}

Todas essas medidas também foram realizadas pela equipe da pesquisa, avaliando-se cada animal individualmente, para um total de 10 cães, pois um deles encontrava-se em policiamento no momento da avaliação. Segundo o escore de condição corporal proposto no protocolo SQ, todos os cães apresentaram condição adequada (escore 2). Porém, se utilizado o sistema de notas mais usual para cães (com 5 pontos), havia um animal considerado magro (escore 2). Na avaliação da condição de tegumento, nenhum cão apresentou qualquer alteração. No momento da avaliação todos os animais apresentaram escore de limpeza 1 (limpo). Também não foram encontrados animais com sinais de claudicação, nem de tosses, bem como evidência de dor ou diarreia. Foi identificado, porém, que uma das fêmeas estava com infecção uterina na semana da avaliação, a qual passou a receber tratamento, sendo o diagnóstico e prescrição do tratamento realizados por médico veterinário responsável.

Quanto aos comportamentos anormais avaliados, $20 \%$ dos cães (2/10) apresentaram estereotipias. Um deles realizou 'andar em círculos' (o animal dava voltas em torno do boxe repetidamente) e o outro, "salto na parede" (o cão dava saltos repetitivos na parede do boxe e, na sequência, girava em torno de seu próprio eixo). Para a reação ao ser humano, $40 \%$ dos cães (4/10) não demostraram sinais de medo ou agressão a curtas distâncias, com postura caracterizada como neutra, amigável/sociável (escore 0), enquanto 20\% (2/10) apresentaram escore 1, mostrando-se atentos, com postura neutra, mas atento/alerta ao avaliador, sem sinal de medo ou agressão. Os 40\% (4/10) restantes apresentaram postura ofensiva, com agressão sem sinais de medo (escore 4).

$\mathrm{Na}$ avaliação individual foi necessária a inclusão de questões relacionadas à socialização dos cães. Primeiramente era questionado sobre a sociabilidade de cada cão (se tolerava a presença de outros cães do canil e se não apresentava comportamento agressivo a outros cães familiares) e em quais momentos havia a oportunidade de terem contato social (exceto policiamento). Foi constatado que apenas um macho não era sociável com os outros cães do canil. Os demais podiam ter contato entre si, o que ocorria em momentos de recreação.

No que diz respeito à avaliação dos estados emocionais dos animais, a ACP gerou três componentes principais com autovalores acima de 1, que juntos explicaram 81,16\% da variação no conjunto de dados. 0 primeiro componente principal apresentou maiores cargas negativas para os termos 'brincalhão', 'busca atenção', 'animado' e 'amigável', e positiva para o termo 'quieto', refletindo um contínuo entre 'brincalhão/ animado - quieto' (Figura 1A). Animais com notas mais baixas nesse fator (cães I e Bi, na Figura 1B) tenderam a apresentar estados mentais mais positivos, segundo a percepção do observador. Por sua vez, no CP2 os termos com maior carga negativa foram 'nervoso', 'agressivo', 'latindo' e 'alerta', e positiva para 'relaxado', expressando um contínuo entre 'nervoso - relaxado'. Animais com menores notas nesse fator foram aqueles que apresentaram expressões relacionadas pelo observador com estados mentais mais negativos (cães E, T, P e K, na Figura 1B). Por fim, no CP3 tiveram altas cargas apenas os termos 'inseguro' $(-0,81)$ e 'confiante' $(0,81)$, podendo refletir a insegurança gerada no animal pela presença do avaliador. 

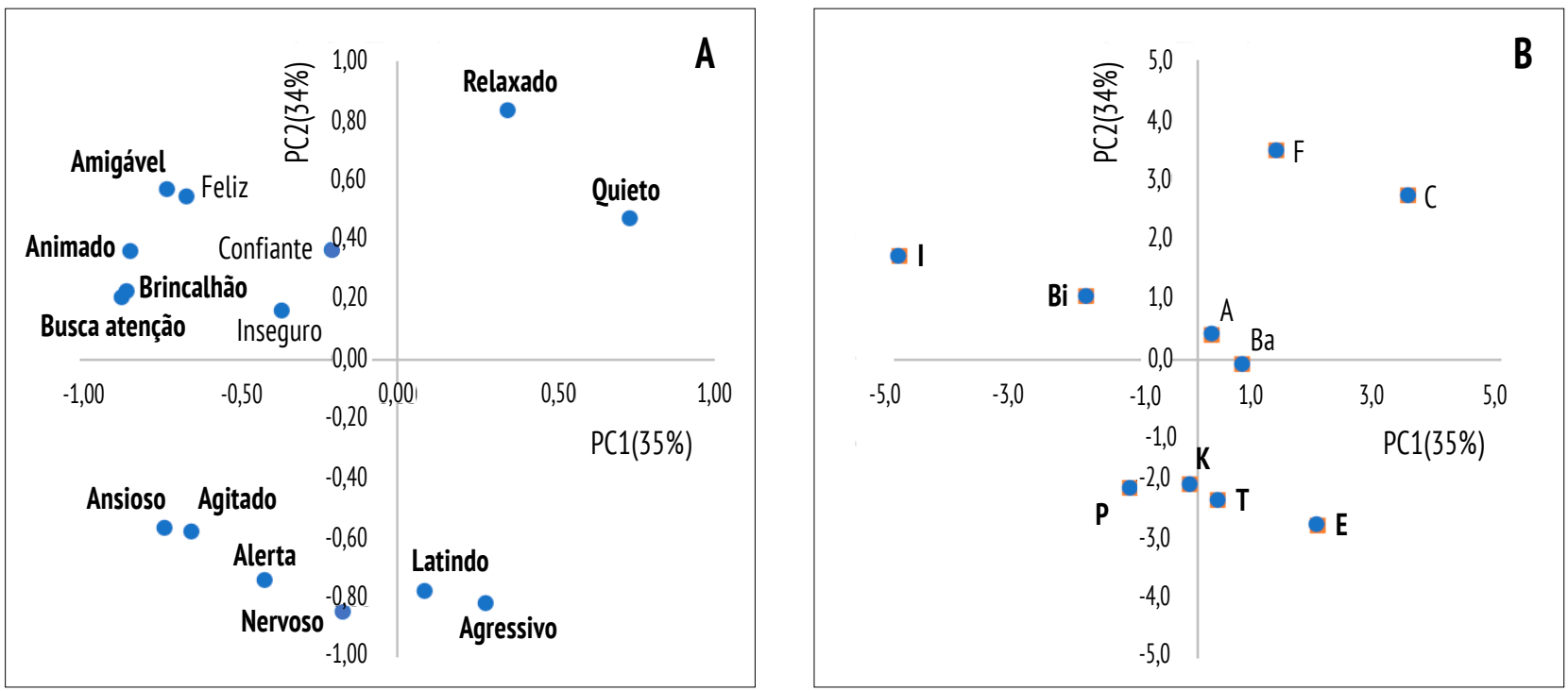

Figura 1 - Resultados da análise de componentes principais aplicada aos termos da avaliação dos estados emocionais dos 10 cães avaliados. A - Gráfico das cargas de cada um dos termos nos dois primeiros componentes principais (CP1 e CP2). Termos em negrito apresentaram as maiores contribuições para a formação de ambos os eixos. B - Gráfico dos escores dos animais nos dois primeiros CP. Letras representam as iniciais dos nomes de cada cão. Nomes em negrito representam animais com estados mentais mais positivos (I, Bi) e negativos (P, K, T, E), segundo a percepção do avaliador.

\section{Rotina dos cães}

A rotina dos cães foi obtida com base em registros diários realizados pelos policiais responsáveis pelos animais. Ao longo das seis semanas do estudo foram solicitadas anotações dos horários e duração das atividades de policiamento, recreação fora dos boxes e treinamento. No que diz respeito ao trabalho em policiamento, oito dos 11 animais estiveram em atividade no período do estudo, sendo que dois não realizaram policiamento por ainda estarem em treinamento e um pela idade avançada. Dentre aqueles que desempenharam trabalhos de policiamento, a carga horária variou de $2 \mathrm{~h}$ (120 min) a 10 h (600 min) de trabalho semanal, com uma média geral de $182 \pm 76 \mathrm{~min} /$ semana. 0 número declarado de saídas para policiamento variou de 0 (nenhuma saída) a 4 saídas semanais. A descrição da carga horária e número de saídas semanais por indivíduo encontra-se na Tabela 3.

Tabela 3 - Carga horária de trabalho semanal (em minutos) realizado pelos oito cães que desempenharam atividades de policiamento ao longo das seis semanas do estudo

\begin{tabular}{lcccccccc}
\hline \multirow{2}{*}{ Período } & \multicolumn{7}{c}{ Cães } \\
\cline { 2 - 9 } & Ba $^{*}$ & $\mathbf{E}$ & $\mathbf{F}$ & $\mathbf{l a}$ & $\mathbf{K}$ & $\mathbf{B i}$ & $\mathbf{P}$ & $\mathbf{T}$ \\
\hline Semana 1 & 0 & 0 & 0 & $60(1)$ & $450(2)$ & 0 & 0 & 0 \\
Semana 2 & 0 & 0 & $360(2)$ & $690(1)$ & $367(4)$ & 0 & $120(1)$ & $300(2)$ \\
Semana 3 & $510(1)$ & $480(1)$ & 0 & 0 & $405(2)$ & 0 & $240(1)$ & $276(3)$ \\
Semana 4 & 0 & 0 & 0 & 0 & $360(1)$ & 0 & $600(1)$ & 0 \\
Semana 5 & $457(2)$ & $300(1)$ & $360(1)$ & 0 & 0 & $300(1)$ & $180(2)$ & 0 \\
Semana 6 & 0 & 0 & $480(1)$ & 0 & $480(1)$ & $360(1)$ & $240(1)$ & $360(1)$ \\
Média (total) & $161(3)$ & $130(2)$ & $200(4)$ & $125(2)$ & $344(10)$ & $110(2)$ & $230(6)$ & $156(6)$ \\
\hline
\end{tabular}

Nota: *Letras representam as iniciais dos nomes de cada cão. Entre parênteses está o número de vezes que o cão saiu para policiamento em cada semana. 
Segundo os responsáveis, a especialidade do cão (faro de entorpecentes ou faro para busca e captura de pessoas) foi o principal fator que contribuiu para variação individual observada nas horas semanais de trabalho, pois cada ocorrência requer tipo específico de funcionalidade.

Quanto à atividade de recreação fora dos boxes, todos os cães do canil estiveram envolvidos, no entanto, com grande variação individual no período de tempo e número de vezes em que foi realizada semanalmente, como pode ser observado na Tabela 4 . Os cães passaram em média $27 \pm 10 \mathrm{~min} / \mathrm{semana}$ em atividades de recreação, variando de 0 (não realizaram qualquer recreação) a 160 minutos/semana.

Sobre o treinamento, foi registrada a realização de 90 min de treinamento com todos os 11 cães na $4^{a}$ semana. Adicionalmente, os cães ' $\mathrm{E}$ ' e 'Bi' passaram por mais 60 min de treinamento na $6^{\underline{a}}$ semana.

Tabela 4 - Carga horária de recreação fora dos boxes (em minutos) realizada pelos 11 cães ao longo das seis semanas do estudo

\begin{tabular}{lccccccccccc}
\hline \multirow{2}{*}{ Período } & \multicolumn{10}{c}{ Cães } \\
\cline { 2 - 11 } & $\mathbf{A}^{*}$ & $\mathbf{B a}$ & $\mathbf{C}$ & $\mathbf{E}$ & $\mathbf{F}$ & $\mathbf{l a}$ & $\mathbf{K}$ & $\mathbf{B i}$ & $\mathbf{P}$ & $\mathbf{T}$ & $\mathbf{I}$ \\
\hline Semana 1 & $60(1)$ & $55(4)$ & $30(4)$ & $60(2)$ & $52(4)$ & 0 & 0 & $40(3)$ & $60(2)$ & $60(2)$ & $60(1)$ \\
Semana 2 & $15(2)$ & $47(3)$ & $47(3)$ & $15(2)$ & $20(2)$ & $20(2)$ & $20(1)$ & $15(2)$ & $40(2)$ & 0 & $25(2)$ \\
Semana 3 & $17(2)$ & $160(2)$ & 0 & $17(2)$ & $17(2)$ & $17(2)$ & $17(2)$ & $17(2)$ & $37(2)$ & $17(2)$ & $17(2)$ \\
Semana 4 & $33(3)$ & $20(4)$ & $30(3)$ & $18(3)$ & $17(4)$ & $20(2)$ & $20(3)$ & $20(4)$ & $20(4)$ & $23(3)$ & $23(3)$ \\
Semana 5 & $18(5)$ & $20(4)$ & $33(6)$ & $20(3)$ & $21(7)$ & $20(6)$ & $34(5)$ & $18(3)$ & $25(6)$ & $15(2)$ & $18(5)$ \\
Semana 6 & $10(1)$ & $20(1)$ & $8(1)$ & $90(1)$ & $20(1)$ & $22(1)$ & $18(1)$ & $13(1)$ & 0 & $36(2)$ & $20(1)$ \\
Média(total) & $25(24)$ & $54(18)$ & $25(13)$ & $37(13)$ & $24(20)$ & $16(13)$ & $18(12)$ & $20(15)$ & $30(16)$ & $25(11)$ & $27(14)$ \\
\hline
\end{tabular}

Nota: * Letras representam as iniciais dos nomes de cada cão. Dentro do parênteses está o número de vezes que o cão realizou recreação fora do boxe em cada semana.

\section{Discussão}

Embora tenha sido desenvolvido para avaliação do bem-estar de cães de abrigo, o protocolo Shelter Quality foi amplamente aplicável à condição do canil militar, sendo necessária a inclusão de alguns indicadores adicionais, como a descrição da rotina de trabalho do cão, por exemplo, para adequação à realidade do canil. Com a inclusão de indicadores adicionais foi possível contemplar o diagnóstico de um maior número de fatores reconhecidamente importantes para o bem-estar dos cães de trabalho militar (Rooney et al., 2009). No que diz respeito à amostragem, o protocolo SQ sugere que as medidas registradas nos animais sejam aplicadas a uma amostra dos cães presentes no estabelecimento, com amostragem mínima de 30 (quando o número de animais alojados for inferior a 60) e máxima de 60 indivíduos (quando o número de cães no abrigo for igual ou superior a 140). No entanto, ao adaptálo para canis militares, considerou-se adequado incluir todos os cães alojados no estabelecimento. Em geral, os canis militares apresentam número total de animais alojados inferior ao de abrigos.

Primeiramente, sobre as medidas para o canil, houve boa aplicabilidade prática para os indicadores presentes no protocolo SQ não requerendo modificações consideráveis. No entanto, foi evidente a necessidade de inclusão de uma avaliação mais refinada sobre a rotina de exercícios, treinamento e recreação fora dos boxes, tendo em vista a importância de tais fatores para o bem-estar dos cães (Rooney et al., 2009). Segundo o protocolo, é apenas registrado na forma de escores que o canil não apresentou rotina regular de atividades fora do boxe. Aconselha-se, no entanto, que seja realizada uma avaliação do tempo e frequência para tais atividades, para todos os animais. Foi proposta, também, uma pergunta a respeito do transporte dos animais, já que este é um fator potencialmente estressante para os cães (Machado, 2013) e importante no contexto de cães militares. 
Além de interferir diretamente no bem-estar, os transportes de média (acima de $3 \mathrm{~h}$ ) e longa duração (acima de $5 \mathrm{~h}$ ) podem causar aumento das concentrações de cortisol salivar, comprometendo a percepção olfativa dos cães, com prejuízos evidentes para atividades como o faro de entorpecentes (Machado, 2013).

Sobre as medidas obtidas nos boxes, o protocolo SQ também se mostrou, de um modo geral, adequado. Segundo a recomendação disponível no protocolo $\mathrm{SQ}$, deve-se dispor de $8 \mathrm{~m}^{2}$ /animal para cães acima de $20 \mathrm{~kg}$, assim, o espaçamento disponível para os cães avaliados $\left(9,80 \mathrm{~m}^{2}\right)$ foi considerado suficiente. Cabe destacar, porém, que embora a maioria dos canis de trabalho militar adotem instalações em boxes individuais, deve-se considerar os benefícios do alojamento coletivo (em duplas) para cães em diferentes circunstâncias de alojamento como abrigos e laboratórios (Hubrecht et al., 1992; Taylor e Mills, 2007). Em um estudo realizado por Hubrecht et al. (1992), quando os cães eram mantidos em alojamento individual era maior a ocorrência de comportamentos mais passivos e de estereotipias, levando-os a propor que o alojamento coletivo pode promover melhores condições de bem-estar por se tratar de animais sociais.

Além disso, a avaliação do tipo de cama esteve de acordo com as recomendações do protocolo SQ sendo considerada adequada por não apresentar pedaços ingeríveis ou enchimento de plástico ou madeira, sem bordas nocivas e evitando o contato direto do animal com o piso. Todavia, este tipo de cama não ofereceria conforto térmico aos animais em situações de baixas temperaturas. Portanto aconselha-se que as avaliações também considerem tal fator relacionado à possibilidade de que o cão se mantenha aquecido durante o inverno. Sugerese que, durante as entrevistas realizadas com o responsável pelo canil, sejam solicitados registros das temperaturas médias, mínimas e máximas no local de alojamento a fim de identificar se é real o risco de estresse térmico durante os meses mais frios e quentes do ano, tendo em conta a raça e porte dos animais alojados.

Ainda com relação às medidas obtidas para os boxes, uma adaptação importante está relacionada à inclusão de um indicador relacionado à disponibilidade de itens de enriquecimento ambiental para os cães. Durante as avaliações realizadas pôdese evidenciar a ausência de enriquecimentos ambientais. Tal fato parece estar associado a uma crença geral de que a provisão de itens de enriquecimento pode piorar o desempenho dos cães no trabalho (Gaines et al., 2008). No entanto, estudos recentes refutam essa hipótese. Segundo trabalho realizado por Gaines et al. (2008), a introdução de itens de enriquecimento alimentar nos canis não causou nenhum efeito prejudicial no desempenho de cães de trabalho, nem na saúde e comportamento desses animais. Com isso, recomenda-se o fornecimento de itens de enriquecimento para melhorar o bem-estar dos cães militares, tais como ossos, brinquedos de morder e kongs (Rooney et al., 2009).

De modo semelhante, as medidas obtidas nos os animais foram aplicáveis aos cães de trabalho militar mediante algumas adaptações descritas a seguir. Para a avaliação da condição corporal, sentiuse a necessidade de um escore com mais notas, que permitisse maior sensibilidade ao detectar as variações existentes na condição corporal dos cães. Por se tratar de animais de trabalho é pouco provável que sejam encontrados animais muito magros ou muito gordos, segundo o sistema de três pontos propostos pelo $\mathrm{SQ}$, que apenas considera como inadequadas condições corporais extremas. Assim, foi utilizado um escore de cinco notas (Hand et al., 2000), onde 1 = muito magro, 2 = magro, $3=$ normal, $4=$ sobrepeso e, 5 = obeso. Esse é o sistema de escore mais usual para a avaliação da condição corporal em cães, o qual possibilitou identificar no presente estudo um animal com escore 2 (magro) e os demais com escore 3 (adequado).

No que diz respeito aos indícios gerais de doenças, não foram encontrados animais com evidência de dor, porém, uma fêmea apresentou infecção uterina no momento da avaliação. 0 diagnóstico foi realizado pelo médico veterinário que realizou o atendimento do animal em clínica especializada. Sinais de doenças não previstos no protocolo, como o corrimento vulvar em fêmeas, por exemplo, podem ser identificados durante a visita, sendo recomendada sua inclusão nos registros a fim de avaliar indícios de outros problemas de saúde não contemplados nas planilhas.

Quanto aos indicadores comportamentais, foi 
possível aplicar todas as medidas sugeridas no protocolo $\mathrm{SQ}$ com algumas adaptações relacionadas a aspectos específicos do comportamento usualmente apresentado pelos cães de trabalho militar. A avaliação da ocorrência de comportamentos anormais foi exequível e informativa, sem a necessidade de qualquer adaptação, sendo registrada a ocorrência de dois cães com condutas repetitivas de andar em círculos e de dar saltos na parede. Esses estão entre os comportamentos repetitivos mais comuns em cães militares e podem expressar baixos níveis de bem-estar como resultado de desafios enfrentados pelos animais ao se adpatarem ao ambiente e à rotina de manejo, por exemplo, um ambiente monótono ou com estímulos aversivos (Haverbeke et al., 2008b).

Foi necessária a inclusão de uma questão que abordasse a socialização dos cães. Foi relatado que apenas um cão não era sociável com outros do canil, havendo oportunidade de contatos entre os demais durante as atividades de recreação. Por se tratar de animais com forte coesão social, é de suma importância o contato com outros indivíduos e com seres humanos durante sua rotina. Segundo Rooney et al. (2009), são consideráveis os benefícios gerados pelo exercício em duplas ou em grupos sociais, como corridas em áreas abertas, pois o contato com coespecíficos atua como um enriquecimento social. Em um estudo realizado na Suíça com cães militares de diversas raças foi demonstrado que os cães com oportunidade de interagir socialmente com outros do mesmo canil, em sessões de $3 \mathrm{~h}$ semanais, durante uma tarde, por oito semanas consecutivas, apresentaram menos respostas comportamentais caracterizadas como defensivas e ofensivas a cães não familiares (Gfrerer et al., 2018). Segundo os autores, mesmo para animais já adultos e alojados individualmente, as sessões de socialização podem melhorar a sociabilidade e, como consequência, a performance do cão no trabalho, fazendo com que o mesmo permaneça mais focado em suas atividades e menos distraído pela presença de outros cães. Para isso, é crucial a observação do comportamento e inspeção regular durante as atividades em grupo a fim de identificar os animais que interagem positivamente entre si, além de intervir em caso de interações agressivas.

Na avaliação relacionada à reação ao ser humano, o escore proposto pelo protocolo SQ buscou avaliar o medo e a agressão dos cães de abrigo. Ambos os fatores são, na maioria das vezes, responsáveis por problemas comportamentais que acabam por prejudicar a interação entre seres humanos e cães, acarretando em diminuição das chances de adoção dos animais. Porém, diferente dos cães de abrigo, cães de trabalho militar apresentam especificidades comportamentais em função do treinamento e das seleções prévias dos animais com base em sua aptidão para o trabalho militar. Animais que têm dificuldade de expressar comportamentos de obediência ou que têm medo ou agressividade excessivos, por exemplo, não são aptos a ingressar nesse tipo de atividade. Portanto, para adequação do escore de avaliação da reação ao ser humano, foi importante a inclusão de uma nota adicional (1 - sem sinal de medo ou agressão e postura atenta/alerta: permanece a uma certa distância e não tenta interagir com o avaliador), pois houve casos em que os animais $(\mathrm{n}=2)$ permaneceram com postura neutra, porém mostrando-se atentos e alertas ao avaliador. Essa reação não estava contemplada no sistema de escores previsto inicialmente no protocolo, onde o animal neutro era descrito como mais relaxado 10 - sem sinais de medo ou agressão e postura neutra: permanece relaxado, olhando para ou ignorando o avaliador, amigável/sociável, se aproxima a curtas distâncias ou tenta interagir com o avaliador).

Segundo trabalho realizado por Haverbeke et al. (2009), a maioria dos cães de trabalho militar apresenta pelo menos uma reação de comportamento agressivo quando expostos a estímulos indutores de agressividade. Da mesma maneira, Haverbeke et al. (2010b) relataram que em torno de $70 \%$ dos cães de trabalho militar avaliados em seus estudos apresentaram comportamentos de agressão relacionada ao medo. Tal reação não foi encontrada nos cães do presente estudo, pois nos casos em que foi observado algum tipo de conduta agressiva $(n=4)$, estas se caracterizaram melhor como 'agressão ofensiva', ou seja, sem sinais de medo.

Em relação à rotina de trabalho dos cães, segundo os registros feitos pelos próprios policiais, foi constatado que nenhuma atividade, como treino, recreação e policiamento, apresentou tempo e frequência padronizados ao longo das semanas do 
estudo. Alguns cães saíram dos boxes de maneira infrequente e irregular, o que, por diversos motivos, pode acarretar prejuízos para o bem-estar desses animais. Primeiramente, o fato de o cão ser privado do contato com seu cuidador por longos períodos de tempo é um sério ponto crítico, capaz de gerar ansiedade nos cães, que em geral desenvolvem apego pelo ser humano com quem interagem regularmente (Ogata, 2016). Além disso, a estabilidade emocional dos cães requer elementos de previsibilidade, o que apenas pode ser garantido por meio de uma rotina regular, com horários e períodos de tempo consistentes para cada uma de suas atividades dentro do canil (Rooney et al., 2009). Por fim, o fato de passar o dia todo dentro do boxe e, em alguns casos, por dias seguidos, é bastante crítico tanto do ponto de vista do estresse psicológico quanto da baixa taxa de exercícios. Foi relatado por Gaines (2008 apud Rooney et al., 2009) que cães de trabalho militar que se exercitam pouco tendem a passar menos tempo em descanso dentro do boxe, apresentam estereotipias, latem mais e visitam o médico veterinário com mais frequência.

Como a manutenção de uma rotina regular de exercícios e de contato regular e frequente com os cuidadores são pontos críticos reconhecidamente importantes para o bem-estar dos cães de trabalho militar, no presente estudo foi incluída uma avaliação mais detalhada da rotina dos cães, onde se registrou o período e a frequência de atividades fora do boxe para policiamento, recreação e treinamento. Um aspecto negativo desse tipo de avaliação é o fato de demandar um acompanha-mento constante da rotina por parte do avaliador ou, alternativamente, ser realizada apenas com base nos relatos e anotações dos próprios responsáveis pelo canil, exigindo o comprometimento dos mesmos com a avaliação. Esse acompanhamento não pode ser obtido facilmente e de modo acurado em um esquema de auditoria simples, realizado durante um único dia de visita. Nesse sentido, destacamos também a importância dos métodos de treinamento como um ponto crítico para o bem-estar e desempenho dos cães em serviço. Apesar de os métodos de treinamento exercerem influência sobre o comportamento, desempenho e bem-estar dos cães de abrigo (Haverbeke et al., 2008a), sua avaliação requer acompanhamento das sessões de treinamento, o que também dificilmente se adequa a um esquema de auditoria com avaliações pontuais. Dessa forma, é recomendável a existência de um programa institucional de manejo do bem-estar dos cães, no qual os dados referentes a um monitoramento permanente do bem-estar dos animais sejam registrados e disponibilizados durante as auditorias.

Em suma, o bem-estar dos cães de trabalho militar depende de diversos fatores relacionados ao animal e ao próprio ambiente. Para sua avaliação é necessária a inclusão de um maior número possível de indicadores que abordem os diversos critérios e pontos críticos de bemestar. A elaboração de esquemas de avaliação pode ser útil não apenas no âmbito científico ou na formulação de regulamentações e instruções normativas baseadas em resultados de pesquisas, mas também em oferecer uma ferramenta prática a gestores de canis, a fim de que identifiquem potenciais riscos ao bem-estar dos animais sob seus cuidados. Por meio da melhoria das condições de manejo e criação dos cães de trabalho militar, espera-se proporcionar a eles melhor qualidade de vida, evitando situações que lhes causem sofrimento. Como consequência, pode-se obter até mesmo melhorias da performance dos mesmos, com aumento da habilidade de aprendizado, maior obediência e atenção durante os exercícios, além da redução do risco de acidentes, como mordidas e ataques aos treinadores e a outros cães.

\section{Conclusão}

Embora tenha sido elaborado para avaliar o bemestar de cães de abrigo, o protocolo SQ foi exequível e prático para a avaliação de cães de trabalho militar. No entanto, para contemplar todas as especificidades desse tipo particular de criação, foi necessário realizar adaptações no protocolo original, com a inclusão de um maior número de indicadores a fim de avaliar elementos relacionados a um maior número possível de pontos críticos de bem-estar. As principais medidas incluídas foram aquelas relacionadas ao transporte dos cães, disponibilidade de enriquecimento ambiental, grau de socialização, rotina detalhada dos cães e métodos de treinamento. Recomenda-se que as avaliações sejam feitas em 
um período maior, podendo, assim, fazer com que o avaliador possa acompanhar de perto a rotina dos animais junto dos responsáveis pelo canil.

Por fim, é necessário aprofundar-se na validação do protocolo adaptado, incluindo um maior número de estabelecimentos avaliados e possibilitando a identificação de todos os possíveis pontos críticos para o bem-estar dos animais. Dessa forma será possível embasar futuras ações que visem a melhoria da qualidade de vida dos cães e dos policiais militares, cuidadores e treinadores, bem como um melhor desempenho dos cães em suas diversas tarefas.

\section{Referências}

Arcuri GB. Efeitos do estresse no manejo reprodutivo em cães machos de trabalho militar [dissertação]. Pirassununga: Universidade de São Paulo; 2015. 58 p.

Baker JL, Miller L. The effects of environmental extremes on working dogs: a collaborative initiative. US Army Med Dep J. 2013:22-7.

Baptista Sobrinho CA, Hatamoto-Zervoudakis LK, Barnabe VH, Nichi M, Oliveira CA. Efeitos do estresse de trabalho sobre parâmetros seminais de cães da raça Rottweiler. Braz J Vet Res Anim Sci. 2009;46(4):280-7.

Barnard S, Pedernera C, Velarde A, Dalla Villa P. Shelter Quality. Welfare Assessment Protocol for Shelter Dogs. Teramo, IT, Girano, ES: Istituto Zooprofilattico Sperimentale dell'Abruzzo e del Molise "G. Carporale", IRTA, Institut de Recerca i Tecnologia Agroalimentàries; 2014.

Beerda B, Schilder MBH, van Hooff JARAM, Vries HW. Manifestations of chronic and acute stress in dogs. Appl Anim Behav Sci. 1997;52(3-4): 307-19.

Broom DM, Fraser AF. Comportamento e Bem-estar de Animais Domésticos. 4 ed. São Paulo: Manole; 2010. $452 \mathrm{p}$.

Burghardt Jr WF. Behavioral considerations in the management of working dogs. Vet Clin Small Anim. 2003;33:417-46.
Diverio S, Barbato O, Cavallina R, Guelfi G, Iaboni M, Zasso $\mathrm{R}$, et al. A simulated avalanche search and rescue mission induces temporary physiological and behavioural changes in military dogs. Physiol Behav. 2016;163:193-202.

Gaines SA, Rooney JN, Bradshaw WJ. The effect of feeding enrichment upon reported working ability and behavior of kenneled working dogs. J Forensic Sci. 2008;53(6):1400-4.

Gfrerer N, Taborsky M, Würbel H. Benefits of intraspecific social exposure in adult Swiss military dogs. Appl Anim Behav Sci. 2018;201:54-60.

Hand MS, Thatcher CD, Remillard RL, Roudebush P. Small animal clinical nutrition. 4 ed. Topeka: Mark Morris Institute; $2000.1192 \mathrm{p}$.

Haverbeke A, De Smet A, Depiereux E, Giffroy JM, Diederich C. Assessing undesired aggression in military working dogs. Appl Anim Behav Sci. 2009;117(1-2):55-62.

Haverbeke A, Diederich C, Depiereux E, Giffroy JM. Cortisol and behavioral responses of working dogs to environmental challenges. Physiol Behav. 2008b;93(1-2):59-67.

Haverbeke A, Laporte B, Depiereux E, Giffroy JM, Diederich C. Training methods of military dog handlers and their effects on the team's performances. Appl Anim Behav Sci. 2008a;113(1-3):110-22.

Haverbeke A, Messaoudi F, Depiereux E, Stevens M, Giffroy JM, Diederich C. Efficiency of working dogs undergoing a new Human Familiarization and Training Program. J Vet Behav. 2010a;5(2):112-9.

Haverbeke A, Rzepa C, Depiereux E, Deroo J, Giffroy JM, Diederich C. Assessing efficiency of a Human Familiarisation and Training Programme on fearfulness and aggressiveness of military dogs. Appl Anim Behav Sci. 2010b;123(3-4):143-9.

Hubrecht RC, Serpell JA, Poole TB. Correlates of pen size and housing conditions on the behaviour of kennelled dogs. Appl Anim Behav Sci. 1992;34(4):365-83. 
Lefebvre D, Depiereux E, Giffroy MJ, Diederich C. Research note: Installation of videorecording equipment had minimal effect on behavioral measurements in 14 kenneled military dogs. J Vet Behav. 2010;5(1):2-6.

Lefebvre D, Diederich C, Delcourt M, Giffroy MJ. The quality of the relation between handler and military dogs influences efficiency and welfare of dogs. Appl Anim Behav Sci. 2007;104(1-2):49-60.

Lefebvre D, Giffroy JM, Diederich C. Cortisol and behavioral responses to enrichment in military working dogs. J Ethol. 2009;27:255-65.

Li Y, Wang GD, Wang MS, Irwin DM, Wu DD, Zhang YP. Domestication of the dog from the wolf was promoted by enhanced excitatory synaptic plasticity: a hypothesis. Genome Biol Evol. 2014;6(11):3115-21.

Machado LLM. Alterações comportamentais e fisiológicas em cães detectores de droga e explosivo após confinamento em caixas de transporte: Influências do estresse no desempenho [dissertação]. Brasília: Universidade de Brasília; 2013. 49 p.
Manly BFJ. Métodos estatísticos multivariados - Uma introdução. 3 ed. Porto Alegre: Bookman; 2008. 229 p.

Ogata N. Separation anxiety in dogs: What progress has been made in our understanding of the most common behavioral problems in dogs. J Vet Behav. 2016;16:28-35.

Rooney N, Gaines S, Hiby E. A practitioner's guide to working dog welfare. J Vet Behav. 2009;4(3):127-34.

Rooney NJ, Gaines SA, Bradshaw JWS. Behavioural and glucocorticoid responses of dogs (Canis familiaris) to kenneling: Investigating mitigation of stress by prior habituation. Physiol Behav. 2007;92(5):847-54.

Schilder MBH, van der Borg JAM. Training dogs with help of the shock collar: short and long term behavioural effects. Appl Anim Behav Sci. 2004;85(3-4):319-34.

Taylor KD, Mills DS. The effect of the kennel environment on canine welfare: A critical review of experimental studies. Anim Welf. 2007;16(4):435-47. 